\title{
Modern Optics and Information Processing
}

\author{
Mikhail M. Miroshnikov \\ S. I. Vavilov State Optical Institute \\ All-Union Research Center \\ St. Petersburg 199034, Russia
}

The continuously accelerating development of optics, optical methods, and optical instruments is typical for the present stage of scientific and technological progress.

The revolution in optics is connected with the discovery of lasers. Along with lasers, optical detectors, considerably improved during recent years, are extensively used in research and development in modern optics.

Optical detectors transform light into electric signals and allow the design of instruments that not only aid the human eye, but provide significant extension of the eye's capability to detect weak and nonvisible fluxes of radiation, thereby aiding in the design of automatic instruments that render production processes automatic.

Infrared imaging (the observation of objects in total darkness by the detection of their thermal radiation) is one of the prominent results of optics research. Infrared imagers, sensitive to a temperature difference of hundredths and thousandths of a degree, make it possible to observe earlier unknown phenomena of nature and discover new potential in such fields as geology, agriculture, medicine, etc., which seem to be far from optics.

Optical instruments transform and register enormous flows of information in the course of measuring distances, angles, and speeds of bodies, and in the study of their shape, etc.

Analysis of the spectra of radiation, absorption, and scattering of light by atoms and molecules gives abundant information about the structure of matter.

The most efficient method of storage, transmission, and transformation of information is an image, which has been used widely by mankind from rock drawings and painting masterpieces to the modern methods of photography, holography, spatial light modulators, and digital processing.

Imaging is the subject of studies in a new area of optics, "iconics," the science devoted to the general properties of images as well as the goals and problems of their transformation, processing, and display, based on various methods and techniques.

One branch of optics that still has future potential is holography, particularly with recording in 3-D media discovered by Y. N. Denisyuk. A hologram as an object's optical equivalent reveals the wonderful property of wave fields to represent material objects. Modern science has only touched on this analogy of a hologram and an object. However, the ability of the 3-D hologram to provide phase conjugation is already the most remarkable achievement of modern laser technology.

In addition to fundamental results, the range of various applications of optics is also increasing.

Optical instruments are used to study the entire universe: Light received by a telescope bares new unexpected information from the heart of the universe. No less surprising are the secrets of living organisms discovered much nearer in studies of the surrounding microcosm by means of a microscope, a simple optical instrument at first glance.

The role of optics is growing in various branches of industry, science, and medicine. In addition to conventional areas (metrology, microscopy, astronomy, etc.), optics is involved in production processes in machine building where 80 to $90 \%$ of all measurements are those of lengths, angles, and inspection of shape and surface roughness.

Laser processing of materials finds a steadily enlarging range of applications. Laser methods of isotope separation are currently used in industry. Large-scale programs of laser thermonuclear fusion are under development.

Optical lithography was originally the basis of microelectronics technology. At present, the problem of submicron resolution for the fabrication of microcircuits of super-large integration is considered on the basis of laser technology and ultraprecision optics.

Optics is introduced in computer science and computer facilities. The use of fiber optic lines in combination with microlasers and optoelectronic components is creating a revolution in communications, drastically increasing the rate of information transmission.

In the early eighties, optical storage disks were put into full-scale production. These disks are the means for digital long-term memory with an extremely high density of recorded information.

Today the use of optics for increasing dramatically computing facilities efficiency with the goal of creating artificial intelligence is one of the most urgent subjects facing the scientific community. 
Modern optical engineering requires fundamentally novel optical materials with unusual combinations of physical and chemical properties. Broad use of laser technology would be impossible without the development and organization of industrial production of activated glass and crystals. Highpower UV and IR lasers in which gas serves as an active medium should be equipped with windows capable of transmitting superintensive light flux without destruction.

The problems in large lens fabrication can be solved only by creating a base of glass with special optical properties and by the development of glass melting and annealing processes that can provide stable series production of highly uniform lenses even up to one meter in diameter. Parameters of glass should not change under any irradiation condition. Creation of a number of so-called athermal glasses is a great success of optical material science. Characteristics of these glasses are kept unchanged within a wide temperature range. Thus they do not require any temperature stabilization system, and in laser systems they ensure small angular divergence of a beam under all operating conditions.

However, even extremely uniform optical glasses cannot solve all the problems. Composite optical materials, e.g., fiber optical components consisting of an enormous number of light guides of about $10 \mu \mathrm{m}$ in diameter, each coated with glass less than $1.0 \mu \mathrm{m}$ thick, provide new potential.

At present, materials are created with the in-depth gradient of the glass refractive index. This permits image formation and light ray control without curvilinear surfaces of lenses, i.e., to change over to planar glass optics.

Modern optics, covering practically all spectral regions, in a number of cases should employ optical components made of crystals.

A desire to develop more efficient technologies other than single crystal growth fostered the creation of optical polycrystal technology or optical ceramics. These materials are produced by the method of vacuum hot compaction of finegrained powders or by their sublimation and recondensation.

Metal optics is a new trend in optical material science that successfully solves three new problems: (1) the production of large lightweight mirrors such as a beryllium substrate covered with a glass mirror reflecting layer, which at the same time protects the environment against highly toxic beryllium; (2) the creation of optical components that can be cooled down to the temperature of liquid gas, which in principle facilitates the reception of weak signals; and (3) the creation of mirrors for high-power laser systems withstanding high-heat loads.

In the field of optical technology, the development of the most recent methods of optical materials processing and the creation of optical coatings for the entire optical spectrum should be noted also.

Ion treatment of optical materials, a new trend in optical technology, has been invented thanks to the State Optical Institute. This invention makes it possible to introduce pro- duction processes for optical elements with a profiled surface of the given microtopography, such as components used in optoelectronics, integrated optics, synthesized holographic components, and phase-only holographic and other diffraction optical components.

Techniques of high-precision dimensional processing and zonal retouching to compensate for the distortion of light wavefronts were developed on the basis of ion treatment, as well as methods for correcting wavefront error of optical systems to $0.01 \lambda$. This improves the quality of optical systems, enhancing their resolution to the diffraction limit.

The use of phase-only synthesized holographic components "kinoform" produced by methods of ion treatment enables the multielement units to be eliminated from optical systems and in such a way to improve lens performance and reduce their weight and size.

The above illustrates the great potential of modern optics and the necessity of further extensive development. A peculiar feature here is that the development of new trends in optics should be combined with the traditional ones, which have not lost their vitality. Moreover, in view of the new possibilities and arising problems, some traditional trends acquire added importance. The investigation of the physiology of vision can serve as an example. To a certain extent, it lost its importance for optics because the visual methods of light detection were replaced by others. However, at present these studies become imperative for the creation of computer vision systems and visual observation in space.

This brief overview shows that today's technical level of a number of the most important programs in science and economy, as well as scientific and technological progress in machine building, metallurgy, chemistry, instrumentation engineering, and the advancement of major trends in medicine depend, to a considerable extent, on the technical level of optical engineering.

Therefore I say that optics and its applications deserve special attention and should be considered as a priority of scientific and technological progress.

The word "optics" was introduced into Russian by Mikhail Lomonosov, an outstanding scientist and patriot of our country. Lomonosov was interested in optics, a science about light and its interaction with matter, during his entire life and especially in his later years. His various achievements in the technology of glass, the basic material of every optical instrument, and in the manufacture of high-quality metal mirrors gained him broad recognition, as well as his invention of the single-mirror off-axis telescope 27 years before Herschel, his creation of the night-vision telescope, the first of its kind in the world, and many other works.

Pushkin wrote, "Respect of the past is the feature that distinguishes the civilization from savagery." Tomention the results obtained by Lomonosov more than 250 years ago is necessary against the background of the achievements of modern optics - for they are an inexhaustible source of new 
ideas and methods of world cognition and development of world resources.

However, before the Great October Socialist Revolution, no advanced manufacturing of optical instruments existed in Russia, nor was there an organized science dealing with light and its interaction with matter, i.e., optical science. No commercial optical glassmaking facilities existed, nor were there any schools for designers of optical systems; there were only individual specialists who were engaged in optical engineering, i.e., the science of instruments based on optics.

The origination of optical science and applied optics in our country was based on the organization of the State Optical Institute (GOI), which was established on the basis of the Physics Institute of Petrography University by its director and then the first director of GOI, Professor Dmitriy Sergeevich Rozhdestvenskiy, an outstanding Russian physicist who later, from 1929, became an active member of the Academy of Sciences of the USSR. Rozhdestvenskiy said, "The history of the Optical Institute is nearly the history of optics in the USSR."

This special section of Optical Engineering, dedicated to optics in our country, to a certain extent is dominated by the work on optical methods and means of information processing.

I would like to express my gratitude to the authors who provided this superb collection of papers and to the referees who reviewed the manuscripts. I would like to thank Editor Brian J.Thompson for inviting me to be the guest editor of this special section and the excellent staff of Optical Engineering who assisted me in accomplishing the many tasks required of a guest editor. My personal thanks to Nina F. Soboleva for her organizational skills and perseverance.

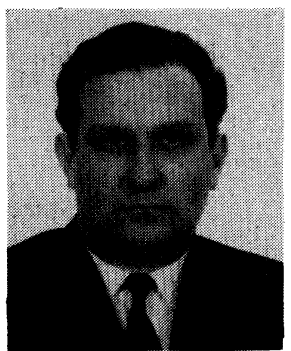

Mikhail Michajlovich Miroshnikov graduated from the Leningrad Institute of Device Engineering for Aviation in 1949 with a radio-engineer certificate. In the same year he began work as a junior researcher at theS.I. VavilovState Optical Institute in the laboratory of A. A. Lebedev, the famous specialist in physical and electronic optics. Miroshnikov has devoted all his research activity to optics and optoelectronic instrument engineering. He has carried out extensive research and development in the areas of optical location, IRengineering, thermovision, metal optics, and iconics. For his outstanding service in the optical science field he was awarded the Hero of the Socialist Labor rank, was elected to be a corresponding member of the USSR Academy of Science, was rewarded the scientific degrees of candidate and doctor of science, and was confirmed in the professor rank in the specialty optoelectronic devices. $\mathrm{He}$ is author of more than 150 scientific proceedings and inventions. Miroshnikov was the director of the S. I. Vavilov State Optical Institute for 23 years. In October 1990, at his request, he became the scientific counselor to the board of directors of the State Optical Institute. 